\title{
ANÁLISIS NO PARAMÉTRICO Y PROYECCIÓN DE SERIES DE PRECIOS DE TABLEROS DE MADERA EN EL MERCADO NACIONAL, CHILE
}

\author{
Toledo, Roger ${ }^{1}$
}

\section{RESUMEN}

Este artículo revisa las opciones metodológicas disponibles en herramientas automatizadas en $R$ para la selección de modelos no paramétricos y la realización de proyecciones. Para ello se han utilizado como ejemplo cuatro series mensuales de precios de tableros de madera para el mercado nacional, y ARIMA y métodos de suavizamiento exponencial para el modelamiento de los datos. Los paquetes y funciones disponibles en $\mathrm{R}$ son más que suficientes para la selección de modelos y la realización de proyecciones, con un amplio conjunto de opciones para el usuario. Sin embargo, para usar estas herramientas se requiere un nivel de usuario intermedio en series de tiempo y R, a fin de permitir una buena interpretación de los resultados, entender las particularidades de las funciones y crear instrucciones sencillas en el software.

Un estudio preliminar de las series determinó que son no estacionarias y ausencia de quiebres estructurales. El modelamiento ARIMA de las series arrojo que el mejor modelo para los datos de tableros contrachapados y MDP es un ARIMA $(0,1,0)$ con constante, mientras que para MDF es un ARIMA $(0,1,0)(0,0,1)_{12}$. En el caso de los modelos de suavizamiento exponencial, los resultados señalan que las series de tableros contrachapados deberían ser modeladas como un ETS $(A, N, N)$, en tanto que las series de MDP y MDF con un ETS $(M, N, N)$. Adicionalmente, los test estadísticos descartan la existencia de tendencias en las series. El análisis de las medidas de precisión para las proyecciones arroja que los modelos ARIMA son más precisos en las series de MDP y MDF, mientras que los modelos de suavizamiento exponencial son más exactos en el caso de los tableros contrachapados.

Palabras clave: ARIMA, métodos de suavizamiento exponencial, proyecciones, $\mathrm{R}$.

\section{SUMMARY}

This article examines the available methodological options in automated tools in $\mathrm{R}$ to select nonparametric models and make forecasts. Four time series for wood-based panels for domestic market were used as examples and ARIMA and exponential smoothing method for data modeling. The packages and functions available in $\mathrm{R}$ are enough to make the model selection and the forecasts, with a wide set of options for the user. However, the use of these tools requires an intermediate user level in time series analysis and $\mathrm{R}$, in order to allow a good interpretation about the results, understand the particularities of the functions and create simple instructions into the software.

A preliminary study about the series confirms that are nonstationary and absence of structural changes. The ARIMA modeling concludes that the best model for plywood and MDP is an $\operatorname{ARIMA}(0,1,0)$ with drift, whereas for MDF is an $\operatorname{ARIMA}(0,1,0)(0,0,1)_{12}$. In the case of exponential smoothing, results indicate that time series of plywood should be modeled as an ETS $(A, N, N)$, and MDP and MDF as an ETS(M,N,N). Additionally, the statistical tests reject the existence of trend in all time series. The analysis of accuracy measures for forecasting shows that whereas that ARIMA models are more accurate for MDP and MDF, for plywood are more precise the exponential smoothing models.

Keywords: ARIMA, exponential smoothing methods, forecasting, R.

\footnotetext{
${ }^{1}$ Instituto Forestal, Chile. roger.toledo@infor.cl.
} 


\section{INTRODUCCIÓN}

Las variables económicas y en particular las series de precios gozan de un cierto interés desde la economía y los negocios debido a lo relevantes que son para el proceso de toma de decisiones de diversa índole. Sin embargo, en el sector forestal no existen instituciones que recopilen y realicen proyecciones de precios u otras variables de tipo económico. Desde el punto de vista empresarial, tampoco se cuenta con indicadores de expectativas específicos. En este escenario, es la propia empresa la que debería realizar sus propias estimaciones para proyectar las variables económicas de interés. Por ello, es de interés importancia dimensionar la dificultad existente en este sentido.

El Instituto Forestal (INFOR) elabora desde hace varias décadas series de precios de productos forestales con altas frecuencias (mensuales, bimensuales o trimestrales), tanto para el mercado interno como para los productos exportados. En estos registros es posible encontrar una buena fuente de información para realizar algunas proyecciones, es pecialmente para los productos más representativos del sector forestal.

En general, las técnicas de estimación de forecasting requieren de al menos un nivel intermedio de conocimientos de econometría y en especial de series de tiempo. Aun con los conocimientos necesarios, el procedimiento para obtener un modelo adecuado requiere invertir un tiempo importante, especialmente si no existe un conocimiento previo de la serie. Además, los recursos necesarios son incrementales en el número de series a analizar. En el caso de los modelos ARIMA (AutoRegressive Integrated Moving Average), ampliamente utilizados, muchos profesionales se inclinan por utilizar la metodología de Box-Jenkins cuya aplicación "es más un arte que una ciencia" (Enders, 2015), apreciación que se relaciona con la subjetividad presente en la selección de modelos que posteriormente pueden ser utilizados para realizar forecasting. Por las razones antes mencionadas, muchos autores han destinado esfuerzos al desarrollo de algoritmos automatizados que asistan en la selección de modelos o que realicen el proceso de forma totalmente automatizada (Hyndman and Khandakar, 2008; Küsters et al., 2006).

Para la realización de forecasting la literatura de series de tiempo ofrece un conjunto de amplio de alternativas univariadas, entre ellos los más conocidos y utilizados son los modelos de suavizamiento exponencial (Exponential Smoothing Models) y los modelos ARIMA (AutoRegressive Integrated Moving Average) (Poler y Mula, 2011). En este contexto, el objetivo de este artículo es revisar el uso de herramientas automatizadas para la selección de este tipo de modelos para su posterior uso en forecasting. Con ello se pretende motivar la introducción de herramientas de forecasting al análisis estadístico y de coyuntura de las series de precios de productos forestales chilenos. Para ello se utilizarán las series de precios de tableros para el mercado nacional expresadas en US $\$ / \mathrm{m}^{3}$, debido a la relevancia del mercado de tableros en el país y a nivel internacional.

Para la evaluación del desempeño de los modelos seleccionados se analizarán los resultados de las estimaciones out-of-sample. Paralelamente, se realizarán revisiones paso a paso para verificar la idoneidad de los modelos obtenidos anteriormente. Como supuestos de trabajo está la selección de modelos más armónicos, los cuales son más adecuados para la realización de forescasting.

Para efecto de las estimaciones se utilizaran el software R (www.r-project.org) y las herramientas automatizadas asociadas a él. Tanto el software como los paquetes estadísticos utilizados son distribuidos bajo una licencia pública general tipo GNU.

En la siguiente sección de materiales y métodos se describen de forma general las metodologías utilizadas, las particularidades de cada una y el procedimiento general de estimación en base a las herramientas de R. También se incluye una descripción de los datos utilizados. Luego, en la sección de resultados se muestran los modelos seleccionados con las herramientas de $R$ y se analiza su desempeño e idoneidad en base a criterios generales. Finalmente se realiza una discusión de los resultados y se plantean las principales conclusiones. 


\section{MATERIALES Y MÉTODOS}

En los últimos 35 años la literatura especializada ha mostrado distintos esfuerzos por desarrollar y revisar software y herramientas u algoritmos que permitieran la selección automatizada o parcialmente automatizada de modelos. Una parte importante de esta literatura es revisada por Küsters et al. (2006), quienes analizan los distintos periodos desde el año 1969 hasta 2006, poniendo énfasis en las limitantes iniciales, los ámbitos de aplicación y los desafíos de estas herramientas a futuro.

Con la proliferación de alternativas metodológicas, la evolución de los software y el interés académico y empírico, ha surgido la necesidad de evaluar y revisar cada una de las opciones relevantes. En este sentido la literatura también es abundante, concentrando gran atención la revisión mediante diversas medidas de ajustes propuestas por diversos autores y las pruebas con grandes grupos de series, tales como las competiciones $\mathrm{M}$, especialmente M3.

En particular, Koning et al (2005) realiza un análisis a un conjunto de 22 métodos, a fin de evaluar su desempeño considerando la significancia estadísticas de sus resultados. Las comparaciones de los autores concluyen que existe un grupo importante de métodos que no son diferentes significativamente, que la complejidad de los mismos no tiene relación con su precisión y que la combinación de métodos no necesariamente genera mejores predicciones.

Dentro de los métodos comparativamente mejor evaluados están Theta, Holt, Dampen y Winter. Un resultado importante del análisis comparativo es que el único método que resulta robusto al variar la periodicidad de los datos y el horizonte de predicción es Theta. En cambio, los demás métodos mencionados muestran drásticos cambios en los resultados dependiendo del contexto.

Por ejemplo, los métodos Holt y Winter son más destacados si se trata de datos mensuales, cualquiera sea el horizonte $(1,6,12$ o 18 meses); por su parte el método Dampen tiene un buen desempeño con datos anuales y trimestrales, pero con datos mensuales y un horizonte de tiempo superior a un periodo es muy pobre.

Hyndman y Billah (2003) realizan una detallada revisión del método Theta, originalmente desarrollado por Assimakopoulos y Nikolopoulos (2000), logrando una importante simplificación algebraica y demostrando que es equivalente a un modelo simple de suavizamiento exponencial (SSE) con pendiente, el cual además es equivalente a un caso especial del método Holt con un parámetro de suavizamiento de la pendiente igual a cero y que puede ser modelado como un proceso $\operatorname{ARIMA}(0,1,1)$ con pendiente.

Adicionalmente, los autores utilizando una competencia M3, con datos anuales, verifican que el modelo SSE con pendiente generalmente se desempeña mejor que el método Theta original y el recalculado por los autores, lo cual se debería al proceso de optimización. Particularmente, el SSE con pendiente obtuvo un peor desempeño comparativo solo en el horizonte de predicción de 3 años, resultado que se explicaría por la gran cantidad de cambios de tendencia en una serie en particular.

Pese a lo anterior, el modelamiento tradicional para realizar forecasting se ha inclinado por los métodos de suavizamiento exponencial o ARIMA. Cada uno de ellos tiene sus ventajas y desventajas. Comparativamente los métodos de suavizamiento exponencial admiten opciones lineales y no lineales, y los casos lineales son todos casos especiales de los modelos ARIMA, mientras que las alternativas no lineales son propias solo de estos modelos. Los métodos de suavizamiento exponencial asumen que las series son no estacionarias, en cambio para los modelamientos con ARIMA no es necesario este supuesto.

Adicionalmente, los modelos de suavizamiento exponencial tienen la limitante de contar con solo 30 modelos en comparación a los modelos ARIMA que ofrecen un mayor número. Pese a ello, Hyndman y Khandakar (2008) sostienen que las alternativas disponibles para los modelos de suavizamiento exponencial son suficientes para adecuarse al comportamiento de casi todas las series económicas y de negocios. Por otra parte, el desempeño de los modelos de suavizamiento 
exponencial es mejor que el de los modelos ARIMA en datos mensuales y trimestrales (Hyndman et al., 2002).

La realización de proyecciones supone la selección de un modelo previamente, ya sea paso a paso o de forma automatizada en base a un algoritmo. En este caso, la atención estará en la selección completamente automatizada en $R$, software que dispone de herramientas que realizan la selección de modelos ARIMA o SARIMA (Seasonal AutoRegressive Integrated Moving Average), en adelante solo ARIMA, y de métodos de suavizamiento exponencial. También permite realizar forecasting con los modelos elegidos.

Estas herramientas están incorporadas en el paquete estadístico llamado "forescast", cuyo autor es Rob James Hyndman. Los output de las proyecciones contienen las predicciones o puntos y los intervalos de confianza para el periodo proyectado.

El paquete forecast también permite obtener estimaciones utilizando el método Theta. Existen además otros paquetes estadísticos complementarios necesarios para análisis adicionales, tales como stats, tseries y urca. Cada uno de estos paquetes tiene amplia documentación en el sitio web de $\mathrm{R}$, la cual es actualizada continuamente.

También es posible consultar la ayuda del software sobre las funciones que se desee utilizar. Por ejemplo, si se desea tener información de las funciones Arima o ets, solo se debe ingresar help(Arima), ?ets o ??ets.

Las automatizaciones que están disponibles en forecast tienen amplias ventajas, especialmente para quién no tiene los conocimientos de un experto o desea comparar sus resultados, ya que permite obtener resultados muy rápidos que pueden acelerar la toma de decisiones y/o alimentar proyecciones multivariadas. Estas ventajas cobran mayor relevancia cuando se necesitan obtener forecasting para grandes set de variables.

Adicionalmente, el paquete forecast permite especificar el modelo ARIMA explícitamente a través de la funcion "Arima" y reutilizar los parámetros obtenidos. Algo similar está disponible para los métodos de suavizamiento exponencial, en el mismo paquete estadístico, a través de la función HoltWinters (también disponible en el paquete stats) y la función ets.

Sin embargo, estas dos opciones no son equivalentes debido a que la función HoltWinters restringe las opciones de modelamiento a un pequeño grupo de modelos con error aditivo, mientras que la función ets establece un conjunto más amplio de posibilidades (30 modelos en total) y considera la opción de un término de error aditivo o multiplicativo.

Esto último es de vital importancia pues entrega mayor flexibilidad en el modelamiento de los datos, sobre todo cuando no se tiene un conocimiento previo que permita tener una adecuada intuición sobre como modelar el término de error.

Sin embargo, es importante considerar que el modelamiento del término de error de forma aditiva o multiplicativa no afecta las proyecciones de la variable, solo tiene un efecto en los intervalos de confianza (Hyndman y Khandakar, 2008).

\section{Forecasting con Modelos ARIMA}

Los modelos AR-I-MA consideran dentro de si los procesos autoregresivos (AR), los integrados (I) y los de media móviles (MA). Adicionalmente existe la opción de que la serie pueda ser ajustada mediante un ARIMA con estacionalidad o SARIMA. forecast.

Toda esta variedad de opciones son consideradas por la función auto.arima del paquete

La nomenclatura utilizada por Hyndman y Khandakar (2008) es la siguiente: 
Donde:

$\varepsilon_{\mathrm{k}}:$
$B$.
$\Phi(z]$ y $\theta(z):$
$\phi(z)$ y $\theta(z):$

m:

Si $\varepsilon \neq 0$, entonces existe un polinomio de orden $d(d+D)$ en la función de predicción del modelo ARIMA sin estacionalidad (con estacionalidad).

Una revisión de los modelos ARIMA se puede encontrar en Enders (2015, cap. 2) o Montgomery et al. (2015, Cap. 5).

Para la selección de modelos ARIMA, un procedimiento común mencionado en la literatura es utilizar la metodología de Box-Jenkins. Según Enders (2015) esta consta de tres grandes pasos: Etapa de identificación, etapa de estimación y una última etapa de chequeo. En la etapa de identificación es donde el investigador analiza la autocorrelación y la autocorrelación parcial. Esta etapa es especialmente útil para identificar posibles series no estacionarias, datos atípicos (outlier) y faltantes. En la etapa de estimación se calculan todos los posibles modelos en base a los resultados de la etapa anterior. Luego se revisan los coeficientes de cada uno de estos modelos, tratando de elegir uno estacionario y parsimonioso que tenga un buen ajuste a los datos. Por último, la etapa de chequeo es donde se revisa que los errores del modelo sigan el comportamiento de un proceso de ruido blanco.

Pese a lo sencillo de la descripción de los pasos a seguir, esta metodología puede llegar a involucrar una gran demanda de tiempo en algunos casos, especialmente cuando se sospecha de quiebres estructurales. La razón de ello está en que los test para raíz unitaria son comúnmente sensibles a los quiebres estructurales o solo admiten un solo quiebre estructural, como el caso del test de Zivot y Andrews (1992). Esto trae consigo que el investigador, ante la evidencia de un posible quiebre, deba testear utilizando varios test. Lo mismo para testear raíz unitaria, no basta con la inspección visual, es necesario realizar preferentemente más de un test estadístico. Así, un proceso meticuloso de revisión de las series puede tomar tiempo, el cual puede ser visto como una inversión en los casos que se planea un trabajo recurrente con dichas series.

Hyndman y Khandakar (2008) establecieron un algoritmo para estimar automatizadamente los modelos ARIMA (con o sin estacionalidad). El algoritmo ha sido implementado a través de la función auto.arima del paquete forecast, la cual permite seleccionar de forma automática todos los valores de los parámetros. También está la opción en el algoritmo para que el usuario establezca algunas limitaciones a los valores de los parámetros. Como se espera, el algoritmo contempla un procedimiento paso a paso que comienza con la elección de los valores para $D$ y $d$. En el caso de $D$, variable binaria en donde cero indica una serie sin estacionalidad, la función auto.arima permite indicar directamente el valor o determinarlo mediante el uso de un test de raíz unitaria estacional como el test OCSB (Osborn-Chui-Smith-Birchenhall) o el test $\mathrm{CH}$ (Casanova-Hansen). Por defecto, si nada se indica, el algoritmo actual utiliza el primer test (OCSB). El test $\mathrm{CH}$ es una versión modificada del original para permitir que m pueda ser $>12$, ya que originalmente solo puede fluctuar en el intervalo $2<m<13$. Luego, para elegir el valor $d$ el algoritmo utiliza el test KPSS propuesto por Kwiatkowski et al. (1992), ya que se prefieren modelos más armónicos, pero también el usuario puede indicar el valor directamente 0 elegir 
alternativamente entre los test Dickey-Fuller Aumentado o Phillips-Perron.

En general, la función auto.arima permite fijar los valores máximos para los parámetros $p_{2} q_{2} P_{3} Q, d$ y $D$. Con estas definiciones la búsqueda se puede restringir, pero en ningún caso esto es necesario pues el algoritmo ya tiene limitaciones por defecto. Así, el usuario puede obviar todo y pedir que la función ajuste de forma completamente automatizada un modelo. En cualquier caso, siempre está la posibilidad de indicar test alternativos para ver posibles cambios en la selección que realiza el algoritmo.

Una vez determinados los valores de $d$ y $D$ ( $\mathrm{m}$ si corresponde), el algoritmo trabaja en función de cuatro modelos básicos, dependiendo si el valor de $m$ es $=1$ ó $>1$. En este paso las selecciones se realizan mediante algún criterio de información; Akaike (AIC), Akaike corregido (AICc) o Schwartz (BIC), que puede ser determinado por el usuario, si nada se indica se utiliza AIC. Luego, en el paso dos se prueban múltiples variaciones del modelo preliminar en base a cambios en los parámetros $(p, q) \circ(P, Q)$, los cuales normalmente varían en conjunto en \pm 1 en cada iteración, a excepción de la constante que puede estar presente o no. Por ello, este paso contempla más de treinta modelos según los valores máximos predefinidos para los parámetros, entre los cuales se selecciona el óptimo en base al criterio cd información definido.

El algoritmo que utiliza la función auto.arima ha establecido algunas restricciones predefinidas para los parámetros, a fin de evitar problemas de convergencia o procesos que se aproximen a un proceso de raíz unitaria. Las restricciones son: a) $(p, q)$ no pueden ser $>5$; b) $(P, Q)$ no pueden ser $>2$; c) todos los modelos con raíces cercanas a la unidad son rechazados ( $<\|1,001\|$; y d) cualquier error en la rutina de optimización implica desechar dicho modelo debido a que los autores asumen que cualquier modelo difícil de ajustar no es un bueno. Finalmente, este algoritmo siempre entregará un resultado válido ya que las posibilidades son finitas y tiene como opción algunos de los cuatro modelos básicos preestablecidos.

La idea de privilegiar modelos más armónicos para la realización de forecasting es una idea que tiene adeptos y detractores (Hyndman y Khandakar, 2008). Sin embargo, nada asegura que un modelo más complejo interprete mejor los datos y permita mejorar las predicciones, en este contexto, puede ser aconsejable pensar en un modelo más simple. Por ello, Enders (2015) sugiere utilizar el criterio de información de Schwartz (BIC) para la selección del modelo final. Esto debido a que BIC castiga en mayor medida que AIC la incorporación de nuevas variables, por lo cual implícitamente opta por modelos menos complejos o más parsimoniosos. Sin embargo, hay que tener presente que BIC es mejor en muestras grandes porque es consistente cuando el número de observaciones tiende a infinito, pero en muestras pequeñas es mejor AIC. Por otra parte, si ambos, BIC y AIC, seleccionan el mismo modelo, entonces se puede tener la seguridad que este es la mejor alternativa. Debido a que AIC tiende a seleccionar modelos sobreparametrizados, es conveniente que al utilizar BIC como criterio se testee si los errores son ruido blanco.

Al trabajar con herramientas automatizadas, es conveniente tener algunas precauciones generales. Antes de cualquier procedimiento es recomendable graficar la serie para detectar cualquier aspecto anormal y revisar las estadísticas descriptivas básicas de la serie. Por lo general, se debe tener presente que los algoritmos pudieran no testear la presencia de quiebres estructurales, como es el caso de la función auto.arima del paquete forecast, o no realizar correcciones ante la presencia de outliers. Por lo anterior, siempre es recomendable realizar una inspección básica inicial a los datos. Además, siempre está la opción de transformar una serie, por ejemplo aplicando logaritmo, para facilitar el análisis, teniendo presente que para las interpretaciones es necesario invertir el proceso y recalcular los intervalos de confianza. Otro aspecto a tener presente es la falta de observaciones y el tratamiento que esto tiene en el software.

Una vez seleccionado el modelo (ARIMA o de suavizamiento exponencial), la función forecast permite realizar forecasting y obtener los respectivos intervalos de confianza. También es posible realizar este procedimiento de dos etapas con una sola instrucción. Por defecto esta función calcula los intervalos con $80 \%$ y $95 \%$ de confianza, pero el usuario puede definir uno o más distintos. Instituciones como el Banco Central de Chile, en su Informe de Política Monetaria, comúnmente utilizan para las gráficas múltiples intervalos basados en niveles de confianza del 
orden del $10 \%, 30 \%, 50 \%, 70 \%$ y $90 \%$. Se debe tener presente que en la medida que el horizonte de predicción se incremente, también lo hará la incertidumbre y con ello el error de predicción. Lo anterior tiene directa relación con los intervalos de confianza, los cuales se amplían conforme crece el horizonte de tiempo. Lo anterior es válido tanto para los modelos ARIMA como para los de suavizamiento exponencial.

\section{Forecasting con Métodos de Suavizamiento Exponencial}

Los modelos de suavizamiento exponencial ofrecen una gran variedad de alternativas para el modelamiento de los datos e incluso de los errores. Montgomery et al. (2015) entregan una extensa revisión del modelamiento utilizando esta metodología y ejemplos utilizando $R$. Por otra parte, Hyndman et al. (2002) proporcionan una extensa revisión del desempeño de varios métodos de selección, especialmente de uno propuesto por los autores que selecciona el mejor modelo dentro de 24 alternativas y que da origen más tarde a la función ets del paquete forecast. En particular, Hyndman et al. (2002) demostraron que el método propuesto para estimar, seleccionar y realizar forecasting de forma automatizada con modelos de suavizamiento exponencial tiene un buen desempeño, especialmente para realizar proyecciones en horizontes de tiempo de no más de 6 periodos con series que presenten estacionalidad.

La cantidad de alternativas o modelos existentes puede ser desagrega de acuerdo a las características de los tres componentes de la serie: Error, tendencia y estacionalidad. El componente estacional puede o no estar presente y, si lo está, entonces puede tener un comportamiento aditivo o multiplicativo. Lo mismo para el componente tendencia, el cual puede tener un comportamiento aditivo, multiplicativo o suavizado. Por último, existe la alternativa de considerar un error aditivo o multiplicativo.

Algo atractivo de la función ets es la posibilidad de modelar el término de error. Según las pruebas realizadas por Hyndman et al. (2002), un modelo con error de tipo multiplicativo fue elegido en 535 casos de 1001 series utilizadas. Esto pone en evidencia que un error de tipo multiplicativo puede ser más frecuente de lo que se cree y, por tanto, la flexibilidad en el modelamiento es necesaria e impone un sesgo hacia las funciones como ets que incorporan esta variante.

Además, Hyndman y Khandakar (2008) señalan que para seleccionar un modelo con error aditivo o multiplicativo es necesario usar algún criterio de información más que medidas de precisión basadas en los errores de las proyecciones a un periodo.

Siguiendo la nomenclatura de Hyndman and Khandakar (2008), los modelos espacio estado para todos los métodos de suavizamiento exponencial pueden ser resumidos en el siguiente sistema de ecuaciones:

$$
\begin{aligned}
& y_{t}=w\left(x_{t-2}\right)+r\left(x_{t-1}\right) \varepsilon_{i} \\
& x_{i}=f\left(x_{t-2}\right)+g\left(x_{t-1}\right) \varepsilon_{i}
\end{aligned}
$$

Donde:

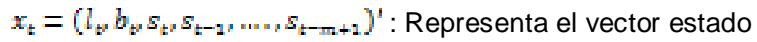

$\varepsilon_{\mathrm{k}}$ Es un proceso ruido blanco con media cero y varianza igual a $\sigma^{z}$ y $\mu_{\mathrm{k}}=w\left(x_{\mathrm{i}-2}\right)$.

Notar que en modelos con error aditivo se tiene que $y_{k}=\mu_{k}+\varepsilon_{k}$ debido a que $r\left(x_{i-1}\right)=1$.

Por otra parte, en modelos con error multiplicativo se tiene que este término es relativo, es decir, $\varepsilon_{\mathrm{k}}=\left(y_{\mathrm{k}}-\mu_{\mathrm{k}}\right) / \mu_{\mathrm{k}}$.

Así se tiene que $r\left(x_{t-1}\right)=\mu_{k}$, e $y_{k}=\mu_{k}\left(1+\varepsilon_{k}\right)$. 
La cantidad de modelos posibles quedan descritos en el Cuadro $\mathrm{N}^{\circ} 1$, donde es posible ver que los 30 modelos disponibles tienen algunas restricciones. En particular, algunas combinaciones de Error, Tendencia y Estacionalidad pueden ocasionar problemas con las estimaciones en aquellos modelos en donde su especificación implica una división por una variable estado (ver modelos en gris). Por otra parte, Hyndman (2014) y Hyndman et al. (2008) señalan que los modelos con errores multiplicativos son estables solo cuando los valores de la variable son estrictamente positivos. Por ello, estos modelos son solo recomendables para variables que excluyan la posibilidad de valores iguales a cero o negativos, como el caso de las series de precios objeto de análisis.

Sin embargo, se debe tener cuidado con el tratamiento para las observaciones faltantes en las bases de datos. En otros casos, solo los modelos con error aditivo y en fondo blanco son los recomendados (Cuadro $\mathrm{N}^{\circ} 1$ ).

Es importante considerar que los modelos planteados en el Cuadro $\mathrm{N}^{\circ} 1$ representan un tipo de modelamiento en particular, pero existen variantes de los mismos. Taylor (2003) hace una revisión de algunas propuestas alternativas para el modelamiento de la tendencia, estableciendo algunas equivalencias, en particular para diferentes valores del parámetro $\phi$.

Comúnmente los parámetros de los modelos $\left(\alpha_{2}, \beta_{i} \gamma_{0} \phi\right)$ fluctúan en el intervalo $(0,1)$ para evitar los casos extremos, a excepción de $\phi$ que debe cumplir la condición $\beta \leq \phi \leq 1$.

Adicionalmente, Taylor (2003) cita que Gadner y McKenzie (1985) sostienen que permitir un $\phi>1$ es una opción peligrosa para procedimientos automatizados de forecasting. Esto se debería a que es poco común encontrar series de tiempo que muestren una fuerte tendencia que aconseje ajustar un modelo con tendencia exponencial.

Una solución para modelar las series que tengan tendencias aparentemente exponenciales es utilizar transformaciones logarítmicas, especialmente si se trata de ajustar un modelo en base a herramientas automatizadas y sin intervención del usuario.

De forma conveniente, para referirse a los modelos del Cuadro $N^{\circ} 1$ se puede seguir a Hyndman (2014), Hyndman et al. (2002) y Taylor (2003), quienes utilizan la sigla ETS (error, tendencia, estacionalidad). Así se tiene que en el Cuadro $N^{\circ} 1$ : ETS $(A, N, N)$ representa un modelo simple de suavizamiento exponencial con error aditivo (celda $(1,1)$ ); ETS $(A, A, N)$ es el método lineal Holt con error aditivo (celda $(2,1))$; ETS $(A, A, A)$ y ETS(M,A,M) son los métodos con tendencia de Holt-Winters con error aditivo y multiplicativo respectivamente; y ETS $\left(A, A_{d}, N\right)$ es el método Holt con tendencia suavizada y error aditivo.

Tal como se señaló antes, la función HoltWinters del software $R$ solo considera algunos modelos con error aditivo, los cuales corresponden a $\operatorname{ETS}\{(A, N, N),(A, A, N),(A, A, A)$, y $(A, A, M)\}$. En particular, esta función estima los valores de los parámetros minimizando el indicador o medida de exactitud MSE $=n^{-1} \sum_{t=-1}^{n}\left(y_{t}-\hat{y_{t}}\right)^{z}$, donde $y_{t}$ representa el valor de la variable en $t$, e $\hat{y_{t}}$ es el valor proyectado de la variable para $t$. Es importante notar que el indicador MSE es escala dependiente.

La función ets tiene algunas características diferenciadoras que la hacen más aconsejable que HoltWinters. Entre otras, la función ets es más flexible ya que admite cualquiera de los modelos planteados, permite ajustar un modelo ya estimado a nuevos datos, optimiza utilizando máxima verosimilitud u otros indicadores, permite obtener intervalos de confianza para las predicciones y arroja valores para los parámetros que son admisibles.

Para la selección de modelos es posible elegir el criterio de información a utilizar (Akaike, Akaike corregido o Schwartz). Adicionalmente, ofrece la opción de restringir la selección a modelos con error aditivo.

ARIMA.

La realización de las proyecciones en $\mathrm{R}$ es similar a la explicado para los modelos 
Cuadro $\mathrm{N}^{\circ} 1$

MODELOS DE SUAVIZAMIENTO EXPONENCIAL

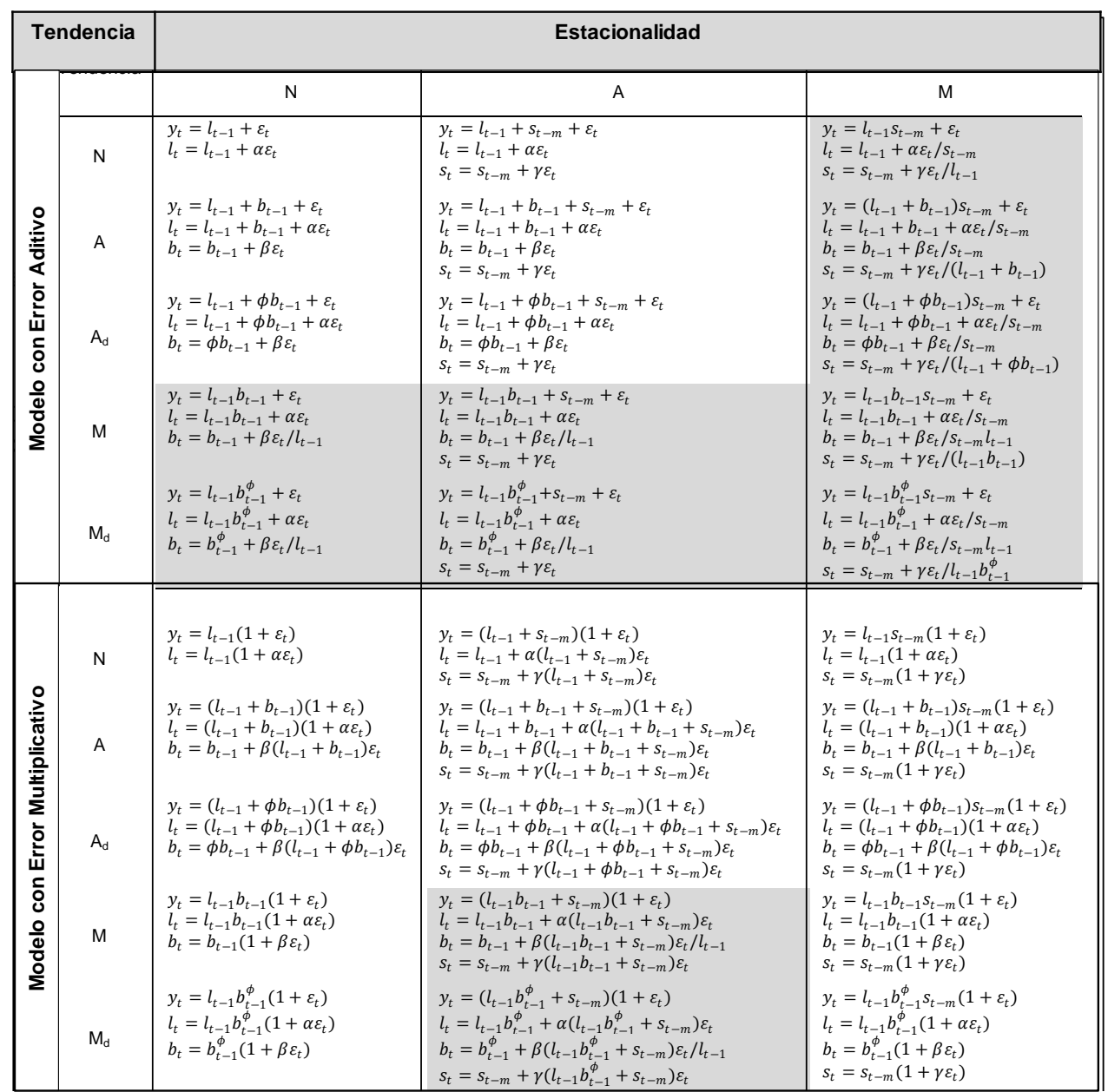

(Fuente: Hyndman, 2014).

T: Tendencia; N: sin tendencia o estacionalidad; $A$ : tendencia o estacionalidad aditiva; $A_{d}$ : Tendencia suavizada; $\mathrm{M}$ : tendencia o estacionalidad multiplicativa; $\mathrm{M}_{\mathrm{d}}$ : tendencia suavizada. Además, $y_{\mathrm{k}}$ es la predicción de la variable para $t$ con la información disponible en $t-1, l_{t}$ es el nivel de la serie en $t, b_{t}$ representa el crecimiento o pendiente de la serie en $t, s_{k}$ es el componente estacional de la serie en $t$, y $m$ denota el número de estaciones por año. Los parámetros $\left(\alpha_{2} \beta_{a} \gamma_{2} \phi\right)$ son constantes.

\section{Datos}

Las series de precios mensuales utilizadas corresponden al mercado nacional, para los tableros MDF (Medium Density Fiberboard) de $15 \mathrm{~mm}$, MDP (Medium Density Particleboard) de $15 \mathrm{~mm}$ y Contrachapados de $9,5 \mathrm{~mm}$ y $18 \mathrm{~mm}$. Las series de tableros contrachapados cubren el periodo enero 2002 a mayo 2015, mientras que los tableros MDF y MDP el periodo enero 2000 a mayo 2015. 
Cuadro $\mathbf{N}^{\circ} 2$

ESTADÍSTICA DESCRIPTIVA SERIES DE PRECIOS TABLEROS DE MADERA MERCADO NACIONAL

\begin{tabular}{|l|r|r|r|r|r|}
\hline \multirow{2}{*}{ Series } & \multirow{2}{*}{ N } & Promedio & Desv. St. & \multicolumn{1}{|c|}{ Min. } & Max. \\
\cline { 3 - 6 } & & \multicolumn{4}{|c|}{$\left(\right.$ US $\left.\$ \mathbf{m}^{3}\right)$} \\
\hline Contrachapado 9,5 mm & 161 & 516,0 & 159,06 & 234,0 & 771,0 \\
\hline Contrachapado 18 mm & 161 & 482,2 & 130,86 & 251,0 & 695,0 \\
\hline MDP 15 mm & 185 & 271,9 & 84,87 & 154,0 & 404,0 \\
\hline MDF 15 mm & 185 & 403,6 & 94,71 & 248,0 & 573,0 \\
\hline
\end{tabular}

Contrachapado $9,5 \mathrm{~mm}$

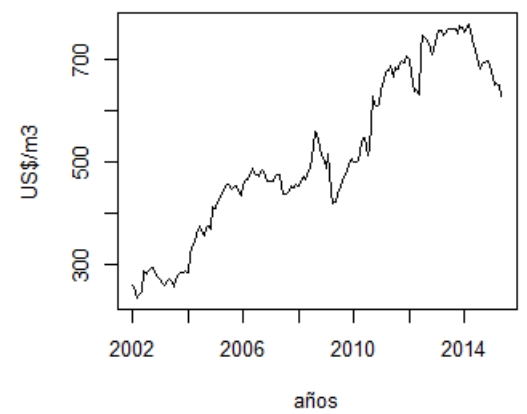

Contrachapado $18 \mathrm{~mm}$

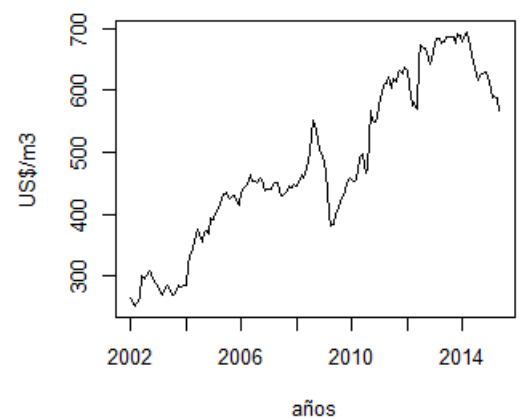

MDP $15 \mathrm{~mm}$

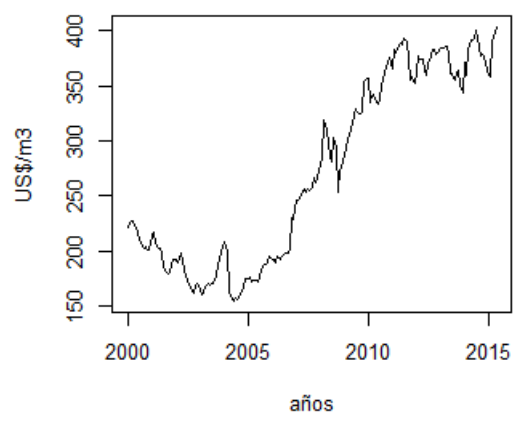

MDF 15mm

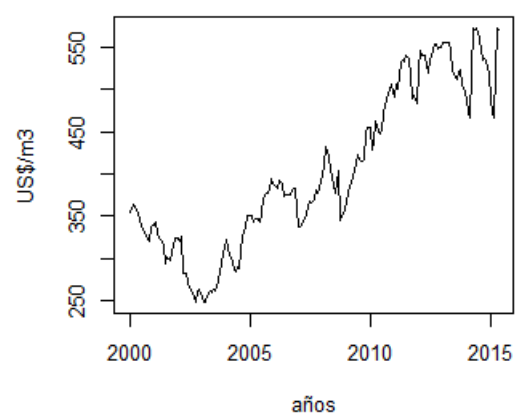

Figura $\mathrm{N}^{\circ} 1$

SERIES DE PRECIOS DE TABLEROS DE MADERA MERCADO NACIONAL

La cantidad de observaciones para cada una de las series de precios son más que suficientes para los requerimientos propios de los modelos que se desea estimar. Se descarta la existencia de datos faltantes o presencia de outliers. El análisis de la descomposición de las series muestra distintas tendencias en la última parte de los periodos. Las series de contrachapados muestran un cambio de tendencia a la baja que parte en el segundo semestre de 2013, por lo que ya se podría hablar de un cambio de mediano a largo plazo. Por su parte, los tableros MDF y MDP mantienen una tendencia al alza en el periodo 2005-2012, pero en los últimos años no existe una clara tendencia. 
Adicionalmente, se comprobó la ausencia de cambios estructurales en las series mediante la realización de los test EFP (Empirical Fluctuation Processes), con las variantes CUSUM y MOSUM, y test F. En todos los casos no fue posible rechazar la hipótesis nula, por lo tanto, se aceptó que no existen quiebres estructurales en las series de precios en primeras diferencias.

\section{RESULTADOS}

El primer paso es determinar si las series son raíz unitaria pese a que visualmente no muestran un comportamiento estacionario (Figura $\mathrm{N}^{\circ} 1$ ). Este paso podría tener implicancias para el modelamiento con métodos de suavizamiento exponencial debido a que estos asumen que las series no son estacionarias. $R$ ofrece varias alternativas de test en diferentes paquetes, entre los que se encuentran ADF (Augmented Dickey-Fuller), PP (Phillips-Perron), KPSS (Kwiatkowski, Phillips, Schmidt and Shin), y Zivot y Andrews.

Los test ADF y PP son bastante similares aunque pueden diferir en su desempeño en muestras pequeñas debido a que abordan la corrección de la correlación serial y la heterocedasticidad en el error de manera distinta. El test KPSS, a diferencia de los dos test antes mencionados, plantea como hipótesis nula que la serie es estacionaria. De forma complementaria está la opción de testear si la serie es estacionaria con un quiebre estructural en un punto desconocido utilizando el test Zivot y Andrews (1992). El quiebre estructural en este test puede estar en el intercepto, la tendencia o ambos.

Es conveniente, cualquiera sea el test que se aplique, tener presente que existen algunas diferencias en los $p$-value dependiendo del paquete y el test particular. También existen diferencias en los métodos seleccionados para el truncamiento en la corrección de la correlación serial, aún para un mismo test en distintos paquetes, lo cual puede tener implicancias para la significancia. Por último, existen test como el KPSS para el cual no es posible obtener los valores de los coeficientes o su significancia en $R$, ya que todas las alternativas disponibles no ofrecen la posibilidad de rescatar dichos valores desde el software. Entre los paquetes utilizados para la realización de test están: tseries, urca y fUnitRoots.

Los resultados obtenidos de los test ADF y PP no son concordantes en todos los casos (Cuadro $\mathrm{N}^{\circ} 3$ ). Por ello se realizó un tercer test, KPSS, el cual incluye constante y tendencia lineal. A partir de este test se determina que las series no son estacionarias, lo cual confirma los resultados del test ADF. Estos resultados permiten asegurar que no existen inconvenientes en la utilización de cualquieras de las dos metodologías propuestas para el modelamiento de las series.

Los resultados del test KPSS (Cuadro $\mathrm{N}^{\circ} 4$ ) fueron obtenidos con un rezago. Si los rezagos en el término de error se incrementan a 4, entonces a excepción de la serie contrachapado de $18 \mathrm{~mm}$ todos los resultados son significativos al $5 \%$. La serie contrachapados de $18 \mathrm{~mm}$ con 4 rezagos solo es significativa al $10 \%$.

Se debe considerar que los niveles de truncamiento para el término de error pueden variar de acuerdo al método utilizado, pero generalmente este se determina en función del número de observaciones. La utilización de cuatro rezagos está de acuerdo al método propuesto por Kwiatkowski et al. (1992), los autores de este test.

Para la evaluación de quiebres estructurales en las series se realizó el test Zivot y Andrews (1992) con las series en niveles y en logaritmo. Para cada una de las series se consideraron distintas hipótesis en la definición del modelo, incorporando dummies para tendencia, intercepto o ambas, tanto para la series en niveles como en logaritmo.

En ningún caso existe evidencia estadística suficiente para rechazar $\mathrm{H}_{0}$, por lo tanto, se acepta que las series son raíz unitaria. En el siguiente cuadro se muestran los resultados para las variables en logaritmo, tal como lo hacen Zivot y Andrews (1992) para series de precios. 
Cuadro $N^{\circ} 3$
TEST DE RAíz UNITARIA PARA LAS SERIES DE PRECIOS DE TABLEROS

\begin{tabular}{|c|c|c|c|c|c|c|}
\hline \multirow{2}{*}{ Test } & \multirow{2}{*}{ Series } & \multicolumn{4}{|c|}{$\Delta y_{t}=a_{0}+\gamma y_{t-1}+a_{2} t+\theta \Delta y_{t-1}+\varepsilon_{t}$} & \multirow{2}{*}{$\begin{array}{c}\text { Raíz Unitaria } \\
\mathbf{H}_{1} \text { : estacionaria } \\
\gamma=0\end{array}$} \\
\hline & & $a_{b}$ & $\gamma$ & $a_{2}$ & $\theta$ & \\
\hline \multirow{8}{*}{ 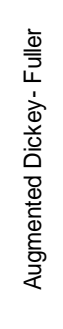 } & \multirow{2}{*}{ Contrachapado $9,5 \mathrm{~mm}$} & 17,88199 & $-0,05248$ & 0,13936 & 0,12149 & \multirow{2}{*}{$-1,7396$} \\
\hline & & $(8,10533)^{\star * *}$ & $(0,03017)^{\star \star}$ & $-0,10411$ & $-0,08185$ & \\
\hline & \multirow{2}{*}{ Contrachapado $18 \mathrm{~mm}$} & 19,66734 & $-0,05937$ & 0,12988 & 0,20861 & \multirow{2}{*}{$-2,0432$} \\
\hline & & $(8,16407)^{* \star *}$ & $(0,02906)^{* * *}$ & $-0,08249$ & $(0.08050)$ & \\
\hline & \multirow{2}{*}{ MDP $15 \mathrm{~mm}$} & 6,85584 & $-0,05307$ & 0,09063 & 0,10282 & \multirow{2}{*}{$-2,7352$} \\
\hline & & $(3,00596)^{\star * *}$ & $(0,01940)^{\star * *}$ & $(0,03095)^{* * *}$ & $-0,07315$ & \\
\hline & \multirow{2}{*}{ MDF $15 \mathrm{~mm}$} & 25,61046 & $-0,10401$ & 0,18672 & 0,07492 & \multirow{2}{*}{$-3,4873^{*}$} \\
\hline & & $(7,98243)^{\star \star *}$ & $(0,02982)^{\star \star \star}$ & $(0,05275)^{\star \star *}$ & $-0,07333$ & \\
\hline \multirow{8}{*}{ 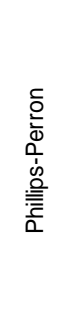 } & I Controphonod 05 & 23,87172 & 0,95806 & 0,10338 & & \multirow{2}{*}{$-7,1979^{\star \star \star}$} \\
\hline & Contracnapado y,5 mm & $-15,142$ & $(0,02934)^{\star * *}$ & $-0,10089$ & & \\
\hline & \multirow{2}{*}{ Contrachapado $18 \mathrm{~mm}$} & 22,14849 & 0,95788 & 0,08044 & & \multirow{2}{*}{$-9,0398^{* * *}$} \\
\hline & & $-13,86864$ & $(0,02874)^{\star \star \star}$ & $-0,08132$ & & \\
\hline & \multirow{2}{*}{ MDP $15 \mathrm{~mm}$} & 13,97683 & 0,95197 & 0,08329 & & \multirow{2}{*}{$-9,3104^{\star \star \star}$} \\
\hline & & $(5,21585)^{\star * *}$ & $(0,01911)^{\star \star \star}$ & $(0,03034)^{\star \star *}$ & & \\
\hline & \multirow{2}{*}{ MDF $15 \mathrm{~mm}$} & 39,03878 & 0,90576 & 0,17006 & & \multirow{2}{*}{$-17,2729^{\star \star \star}$} \\
\hline & & $(11,69950)^{* * *}$ & $(0,02896)^{\star * *}$ & $(0,05119)^{\star * *}$ & & \\
\hline
\end{tabular}

(Fuente: Elaboración propia)

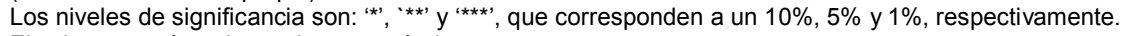

El valor en paréntesis es el error estándar.

El test ADF incluyó en la regresión la variable dependiente rezagada en un periodo.

De acuerdo a Phillips y Perron (1988) el término $a_{0}=\tilde{\mu}, a_{z}=\beta$, y $t=\left(t-\frac{\pi}{z}\right)$ en el test PP.

\section{Cuadro $\mathrm{N}^{\circ} 4$}

TEST KPSS

\begin{tabular}{|c|c|}
\hline Series & $\mathbf{H}_{0}$ : Serie Estacionaria \\
\hline Contrachapado 9,5 mm & $0,3384^{* * *}$ \\
\hline Contrachapado $18 \mathrm{~mm}$ & $0,3022^{* * *}$ \\
\hline MDP $15 \mathrm{~mm}$ & $1,1679^{* * *}$ \\
\hline MDF $15 \mathrm{~mm}$ & $0,7875^{\star * *}$ \\
\hline
\end{tabular}

Los resultados de la utilización de la función auto.arima se muestran en el Cuadro $\mathrm{N}^{\circ} 6$. Según éstos, excepto la serie de precios de los tableros MDP, todas las series debieran ser modeladas como un proceso ARIMA $(0,1,0)$ con constante. Complementariamente se realizaron test de raíz unitaria estacionales, como $\mathrm{CH}$ y OCSB, los cuales rechazan la necesidad de alguna diferenciación, es decir $D=0$. Esto lo verifica la función auto.arima internamente pero puede también ser verificado paso a paso con la función nsdiffs. 
El análisis de la autocorrelación y la autocorrelación parcial, realizado de forma complementaria, no arroja indicios que las series puedan ser modeladas como modelos ARMA, de acuerdo a los patrones de selección de Enders (2015). A diferencia de las demás series de precios, la autocorrelacon parcial en tableros MDF indica que se debe explorar un modelamiento con un término de error estacional, es decir $Q=1$. Esto ya que la autocorrelación cae lentamente (sin oscilaciones) y la autocorrelación parcial no tiene sus primeros elementos significativos, pero si algunos retardos posteriores, lo cual indica que existe información que se podría modelar.

\section{Cuadro $\mathrm{N}^{\circ} 5$}

TEST ZIVOT \& ANDREWS PARA LAS SERIES DE PRECIOS DE TABLEROS

\begin{tabular}{|c|c|c|c|c|c|c|c|}
\hline \multirow{2}{*}{ Series } & $\mathrm{T}_{\mathrm{B}}$ & ax & $a$ & 8 & $\theta$ & $y$ & $\mathbf{H}_{0}$ \\
\hline & \multicolumn{7}{|c|}{ Modelo general } \\
\hline \multirow{2}{*}{ Contrachapado $9,5 \mathrm{~mm}$} & \multirow{2}{*}{25} & 0,5368 & 0,9039 & 0,0004 & 0,03785 & $-0,00002$ & \multirow{2}{*}{$-2,9108$} \\
\hline & & $(0,1841)^{\star \star \star}$ & $(0,0330)^{\star \star \star}$ & $-0,0011$ & $(0,01829)^{\star \star \star}$ & $-0,0011$ & \\
\hline \multirow{2}{*}{ Contrachapado $18 \mathrm{~mm}$} & \multirow{2}{*}{25} & 0,5004 & 0,9127 & $-0,0004$ & 0,0389 & 0,00065 & \multirow{2}{*}{$-2,807$} \\
\hline & & $(0,1754)^{\star \star \star}$ & $(0,0311)^{\star \star \star}$ & $-0,001$ & $(0,0166)^{\star \star \star}$ & $-0,001$ & \\
\hline \multirow{2}{*}{ MDP 15 mm } & \multirow{2}{*}{82} & 0,5704 & 0,8904 & 0,00003 & 0,0541 & 0,00029 & \multirow{2}{*}{$-3,6804$} \\
\hline & & $(0,1581)^{\star \star \star}$ & $(0,02978)^{\star \star \star}$ & $-0,0002$ & $(0,01612)^{\star \star \star}$ & $-0,0003$ & \\
\hline \multirow{2}{*}{ MDF $15 \mathrm{~mm}$} & \multirow{2}{*}{44} & 0,9355 & 0,8406 & $-0,0014$ & 0,0478 & 0,0021 & \multirow{2}{*}{$-4,1165$} \\
\hline & & $(0,2297)^{\star \star \star}$ & $(0,0387)^{\star \star \star}$ & $(0,0006)^{\star \star \star}$ & $(0,0152)^{\star \star \star}$ & $(0,0007)^{\star \star \star}$ & \\
\hline
\end{tabular}

Nota: $T_{\mathrm{B}}$ es el periodo en que ocurre el probable cambio estructural. El valor en paréntesis es el error estándar.

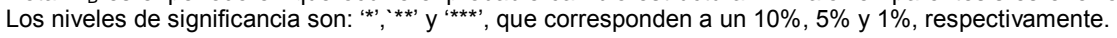

\section{MODELOS SUGERIDOS POR AUTO.ARIMA}

\begin{tabular}{|l|l|c|r|}
\hline \multirow{2}{*}{ Serie } & \multicolumn{3}{|c|}{$\begin{array}{c}\text { Proceso Automatizado } \\
\text { (función auto.arima) }\end{array}$} \\
\cline { 2 - 4 } Contrachapado $9,5 \mathrm{~mm}$ & Modelo Elegido & BIC & Log > Likelihood \\
\hline Contrachapado $18 \mathrm{~mm}$ & ARIMA $(0,1,0)$ con constante & 1363,03 & -694.84 \\
\hline MDP $15 \mathrm{~mm}$ & ARIMA $(0,1,0)$ con constante & 1362,04 & -676.44 \\
\hline MDF $15 \mathrm{~mm}$ & ARIMA $(0,1,0)(0,0,1)_{12}$ & 1599,83 & $-675,8$ \\
\hline
\end{tabular}

En el siguiente cuadro se muestran las opciones seleccionadas por la función ets para el modelamiento de las series de acuerdo a métodos de suavizamiento exponencial. Como se observa, todas las series son modeladas sin tendencia y sin estacionalidad. Esto último, establece una consistencia con lo que ya el paquete forecast determinó anteriormente con la función auto.arima y lo verificado de forma complementaria. Por otra parte, las series de contrachapados tienen un error aditivo y las series de tableros MDP y MDF tienen un término de error multiplicativo.

Al igual que para el caso de los modelos ARIMA, se seleccionó el criterio BIC para la selección. Sin embargo, se debe señalar que esta última elección no tuvo efectos prácticos para ninguna de las selecciones realizadas con respecto a las series analizadas, lo cual confirma que se ha seleccionado la mejor alternativa posible. Todas las demás opciones son las que por defecto tiene el algoritmo de la función ets. 
La selección realizada por ets indica que el mejor modelo posible para las series es uno que mantiene el valor presente como pronóstico para el siguiente periodo. Esto es conocido como el naive method, el cual es un caso extremo cuando $\alpha=1$. Se debe tener presente que el algoritmo de ets reporta por defecto $\alpha=0,9999$ en estos casos, no 1 , ya que se ha restringido que este parámetro sea $<1$. Esto no es reportado en el material disponible de la función o el paquete. Por otra parte, se debe tener presente también que el modelamiento del error de forma multiplicativa o aditiva no tiene implicancias para la predicción de la variable, como ya se mencionó, solo afecta la amplitud del intervalo de confianza. En otras palabras, si el interés es solo conocer el punto de predicción sin su intervalo de confianza, un modelo $E T S(A, N, N)$ o ETS $(M, N, N)$ reportará los mismos resultados. Desde el punto de vista de modelamiento estos dos métodos son los más simples y los menos informativos. Una de las explicaciones posibles para la elección de estos modelos es que los datos tendrían una media a la cual regresan lentamente, lo cual justificaría la ausencia de tendencia. Adicionalmente, la estacionalidad está descartada por los test CH y OCSB.

Cuadro $\mathrm{N}^{\circ} 7$

MODELOS SUGERIDOS POR ETS

\begin{tabular}{|l|c|c|c|}
\hline \multirow{2}{*}{ Series } & \multicolumn{3}{|c|}{$\begin{array}{c}\text { Proceso Automatizado } \\
\text { (función ets) }\end{array}$} \\
\cline { 2 - 4 } & Modelo Elegido & \multicolumn{1}{|c|}{ BIC } & $\sigma_{s}$ \\
\hline Contrachapado $9,5 \mathrm{~mm}$ & ETS(A,N,N) & 1771,35 & 18,71 \\
\hline Contrachapado $18 \mathrm{~mm}$ & ETS $(\mathrm{A}, \mathrm{N}, \mathrm{N})$ & 1733,80 & 16,65 \\
\hline MDP $15 \mathrm{~mm}$ & ETS(M,N,N) & 1796,51 & 0,04 \\
\hline MDF 15 mm & ETS(M,N,N) & 1996,70 & 0,04 \\
\hline
\end{tabular}

La optimización de modelos en ets se realiza a través del log-likehood o por indicadores elaborados desde los errores, como MAE, MSE, AMSE o Sigma (desviación estándar de los errores). Por defecto, ets selecciona log-likehood, opción utilizada en este caso. La selección de modelos solo se realiza en base a los criterios de información (AIC, AICc, BIC).

Es conveniente tener presente que los criterios de información calculados para los modelos ARIMA y ETS no son comparables. Adicionalmente, en el caso de los modelos ARIMA los criterios de información no son comparables para diferentes niveles de diferenciación (Hyndman y Khandakar, 2008). También pueden existir diferencias en los criterios de información para un mismo modelo en diferentes software, esto porque el condicionamiento en la función de verosimilitud puede ser distinto o se puede haber omitido o incluido una constante. Sin embargo, al utilizar el mismo software para todo el proceso de estimaciones estas observaciones no tienen efectos para la selección de modelos pero si para la comparación.

Para medir la calidad del ajuste en el forecasting out-of-sample $\mathrm{R}$ ofrece la posibilidad de obtener medidas de precisión utilizando la función accuracy, entregando una gran variedad de indicadores ampliamente utilizados y discutidos en Hyndman y Koehler (2006). Según Hyndman y Koehler (2006) MASE (Mean Absolute Scaled Error) es preferible para comparar series en diferentes escalas o que tienen valores cercanos a cero o negativos; MAE (Mean Absolute Error) es más simple de interpretar cuando todas las series están en la misma escala, es menos sensible que otras medidas escala dependientes a la presencia de outlier y es útil cuando se analizan diferentes modelamientos para un mismo set de datos; y MAPE (Mean Absolute Percentage Error) es el mejor indicador cuando los valores de la series son positivos y para comparar el desempeño de las proyecciones utilizando diferentes conjuntos de datos. De acuerdo a estas conclusiones, todas estas medidas de precisión son idóneas para series de precios $(p \gg 0)$ expresadas una misma moneda, pero se debe tener precaución en la comparación de resultados en base a datos expresados en términos nominales y reales. Esto no descarta el uso de otras medidas construidas en términos relativos, algo reportado en Hyndman y Koehler (2006). 
Para el análisis es necesario considerar que MAE, MAPE y MASE son medidas donde las diferencias son consideradas en valor absoluto. Por otra parte, solo MAPE y MASE son escala dependientes, lo cual es importante cuando se trata de series de precios referidas a diferentes unidades de medida. Además, MASE se debe interpretar en torno a 1, es decir, un valor $<1(>1)$ indica que el modelo evaluado entrega, en promedio, un menor (mayor) error en comparación con el pronóstico basado en el naive method.

Según Montgomery et al. (2015) para revisar la calidad de los ajustes de los modelos se puede dividir la muestra en dos partes, una para realizar el ajuste y la otra para testear el desempeño del modelo elegido. Para ello se dividieron las series en dos partes: la primera incluyó aproximadamente el $90 \%$ de las primeras observaciones y la segunda quedó conformada por las últimas observaciones. De esta forma, la segunda submuestra para las series de precios de contrachapados está compuesta por las últimas 16 observaciones, mientras que para las series de MDP y MDF esta contiene 18 datos.

Utilizando la función auto.arima se ajustó un modelo automáticamente a los datos de la submuestra inicial (in sample) y luego se realizaron dos pasos paralelos. El primero fue utilizar dichos parámetros para ver el ajuste en los datos de la segunda submuestra (modelo "extendido").

El segundo paso fue obtener un forecasting para el periodo cubierto por la segunda submuestra (out-of-sample) desde el modelo ajustado automáticamente para los datos iniciales, y obtener medidas de ajuste a partir del análisis de las proyecciones versus los datos reales.

Se debe tener presente que el horizonte de tiempo utilizado en el análisis out-of-sample debe ser común para los análisis comparativos debido a que este afecta los resultados de las medidas de precisión.

Los resultados de los procedimientos antes mencionados, utilizando modelamiento tipo ARIMA y ETS, son reportados en los Cuadros $\mathrm{N}^{\circ} 8$ y 9 . Todos los modelos de forecasting dentro de la muestra (in sample) tienen un MASE prácticamente igual a 1, lo cual está relacionado al tipo de modelos seleccionados y su relación con la base de comparación (naive method).

En el análisis comparativo del desempeño out-of-sample de los modelos ARIMA y ETS se aprecia que las proyecciones en base a los modelos de suavizamiento exponencial entregan una mayor precisión para las series de contrachapados, en cambio para MDF y MDP los modelos ARIMA tienen una mayor exactitud.

Cuadro $\mathrm{N}^{\circ} 8$

MEDIDAS DE AJUSTE MODELO ARIMA

\begin{tabular}{|l|l|r|r|r|}
\hline \multirow{2}{*}{ Serie } & \multirow{2}{*}{ Modelo } & \multicolumn{3}{|c|}{ Medidas de Ajuste } \\
\cline { 2 - 5 } & & \multicolumn{1}{|c|}{ MAE } & \multicolumn{1}{|c|}{ MAPE } & \multicolumn{1}{c|}{ MASE } \\
\hline \multirow{3}{*}{ Contrachapado 9,5 mm } & Extendido & 13,16 & 1,91 & 1,07 \\
\cline { 2 - 5 } & In sample & 11,99 & 2,54 & 0,98 \\
\cline { 2 - 5 } & Out-of-sample & 88,44 & 13,16 & 7,23 \\
\hline \multirow{3}{*}{ Contrachapado 18 mm } & Extendido & 11,66 & 1,88 & 1,07 \\
\cline { 2 - 5 } & In sample & 10,76 & 2,38 & 0,98 \\
\cline { 2 - 5 } & Out-of-sample & 77,79 & 12,83 & 7,10 \\
\hline \multirow{3}{*}{ MDP 15 mm $15 \mathrm{~mm}$} & Extendido & 20,43 & 3,78 & 0,97 \\
\cline { 2 - 5 } & In sample & 8,87 & 2,34 & 0,99 \\
\cline { 2 - 5 } & Out-of-sample & 36,94 & 6,99 & 4,12 \\
\hline & Extendido & 9,42 & 2,48 & 0,94 \\
\cline { 2 - 5 } & In sample & 6,05 & 2,40 & 0,99 \\
\cline { 2 - 5 } & Out-of-sample & 22,67 & 5,84 & 3,70 \\
\hline
\end{tabular}


Cuadro $\mathrm{N}^{\circ} 9$

MEDIDAS DE AJUSTE MODELO ETS

\begin{tabular}{|l|l|r|r|r|}
\hline \multirow{2}{*}{ Serie } & \multirow{2}{*}{ Modelo } & \multicolumn{3}{|c|}{ Medidas de Ajuste } \\
\cline { 2 - 5 } & & MAE & \multicolumn{1}{|c|}{ MAPE } & \multicolumn{1}{c|}{ MASE } \\
\hline \multirow{3}{*}{ Contrachapado 9,5 mm } & Extendido & 11,56 & 1,68 & 0,94 \\
\cline { 2 - 5 } & In sample & 12,19 & 2,59 & 1,00 \\
\cline { 2 - 5 } & Out-of-sample & 60,63 & 9,01 & 4,96 \\
\hline \multirow{3}{*}{ Contrachapado 18 mm } & Extendido & 10,27 & 1,65 & 0,94 \\
\cline { 2 - 5 } & In sample & 10,88 & 2,40 & 0,99 \\
\cline { 2 - 5 } & Out-of-sample & 54,38 & 8,96 & 4,97 \\
\hline \multirow{3}{*}{ MDF 15 mm } & Extendido & 20,11 & 3,71 & 0,95 \\
\cline { 2 - 5 } & In sample & 8,91 & 2,35 & 0,99 \\
\cline { 2 - 5 } & Out-of-sample & 39,94 & 7,45 & 4,46 \\
\hline & Extendido & 9,47 & 2,49 & 0,95 \\
\cline { 2 - 5 } & In sample & 6,10 & 2,41 & 0,99 \\
\cline { 2 - 5 } & Out-of-sample & 29,39 & 7,59 & 4,79 \\
\hline
\end{tabular}

\section{DISCUSIÓN Y CONCLUSIONES}

Ambos tipos de modelamiento, ARIMA y métodos de suavizamiento exponencial, son buenas alternativas y se complementan para la realización de forecasting. Sin embargo, la utilización de los modelos de suavizamiento exponencial requiere verificar que las series no sean estacionarias, lo cual implica que el usuario debe tener conocimientos necesarios respecto de los test y el uso de un software estadístico. Adicionalmente, el usuario debe estar en condiciones de conducir una revisión inicial de las series a fin de descartar la presencia de datos faltantes y outlier en $\mathrm{R} u$ otro software. Esto se debe a que las herramientas automatizadas del paquete forecast no tienen un control sobre tales situaciones.

Un hecho relevante para el manejo de $\mathrm{R}$ es que no existen outputs estandarizados para los test citados. Esto conlleva que se deban revisar todas las alternativas existentes en los diferentes paquetes para un mismo test, a fin de conocer las diferencias. Por otra parte, existen aparentemente tests que están disponibles en diferentes paquetes del software, pero que solo se diferencian en el output, debido a que el test se corre en un solo paquete, cosa que muchas veces los autores no mencionan en el documento disponible para el paquete respectivo o test. Los problemas más comunes son la falta de los valores críticos correspondientes y/o la indicación del nivel de significancia y la omisión de los parámetros con su error estándar y/o el nivel de significancia.

Al margen de los resultados específicos obtenidos para las series bajo análisis, las herramientas automatizadas entregan una rápida respuesta al modelamiento de las series, con un procedimiento que entrega algunas flexibilidades para el usuario. Por ejemplo, el usuario puede fijar ciertos valores de parámetros, elegir el horizonte de tiempo, elegir el criterio de información para la selección de modelos y reutilizar los parámetros estimados y aplicarlos a nuevos datos. Por otra parte, los outputs entregan información sobre los criterios de información, los resultados de varias medidas de precisión basadas en los errores, los puntos de estimación, los intervalos de confianza y la opción de graficar de forma complementaria las proyecciones y las desviaciones correspondientes a los niveles de confianza.

Por último, queda de manifiesto que el uso de las herramientas automatizadas de R, tanto para el modelamiento como para forecasting, requieren de un nivel intermedio de conocimiento en series de tiempo. Además, es necesario que el usuario tenga conocimientos intermedios en $\mathrm{R}$, al menos suficientes para realizar e interpretar test estadísticos y escribir instrucciones simples. 


\section{REFERENCIAS}

Enders, W., 2015. Applied Econometric Time Series (Fourth Edition). US: Wiley.

Hyndman, R. J., 2014. Forecasting: Principles \& Practice. University of Western Australia.

Hyndman, R. J. and Billah, B., 2003. Unmasking the Theta Method. International Journal of Forecasting, vol. 19, pp. 287-290.

Hyndman, R. J. and Koehler, A. B., 2006. Another Look at Measures of Forecast Accuracy. International Journal of Forecasting, vol. 22, pp. 679-688.

Hyndman, R. J. and Khandakar, Y., 2008. Automatic Time Series Forecasting: The Forecast Package for R. Journal of Statistical Software, vol. 27 (3), pp. 1-22.

Hyndman, R. J.; Akram, M. and Archibald, B. C., 2008. The Admissible Parameter Space for Exponential Smoothing Models. Annals of the Institute of Statistical Mathematics, 407-426.

Hyndman, R. J.; Koehler, A. B.; Snyder, R. D. and Grose, S., 2002. A State Space Framework for Automatic Forecasting Using Exponential Smoothing Methods. International Jounal of Forecasting, vol 18, pp 439-454.

Koning, A. J.; Franses, P. H.; Hibon, M. and Stekler, H. O., 2005. The M3 Competition: Statistical Test of the Results. International Journal of Forecasting, vol. 21, pp. 397-409.

Küsters, U.; McCullough, B. and Bell, M., 2006. Forecasting Software: Past, Present and Future. International Journal of Forecasting, vol. 22, pp. 599-615.

Kwiatkowski, D.; Phillips, P. C.; Schmidt, P. and Shin, Y., 1992. Testing the Null Hypothesis of Stationarity Against the Alternative of a Unit Root. Journal of Econometrics, vol. 54, pp. 159-178.

Montgomery, D. C.; Jennings, C. L. and Kulahci, M., 2015. Introduction to Time Series Analysis and Forecasting (Second Edition). US: Wiley.

Phillips, P. C. and Perron, P., 1988. Testing for a Unit Root in Time Series Regression. Biometrika, vol. 75(2), pp.335-346.

Poler, R. and Mula, J., 2011. Forecasting Model Selection Through Out-of-sample Rolling Horizon Weighted Errors. Expert Systems with Applications, vol 38, pp. 14.778-14.785.

Taylor, J. W., 2003. Exponential Smoothing with a Damped Multiplicative Trend. International Journal of Forecasting, vol. 19, pp. 715-725.

Zivot, E. and Andrews, D. W., 1992. Further Evidence on the Great Crash, the Oil-price shock, and the Unit-root Hypothesis. Journal of Business \& Economic Statistics, vol. 10 (3), pp. 251-270. 